\title{
Soft Tissue Sarcomas-The Pitfalls in Diagnosis and Management!!
}

\author{
Chintamani
}

Received: 15 February 2012 / Accepted: 22 February 2012 /Published online: 10 March 2012

(C) Indian Association of Surgical Oncology 2012

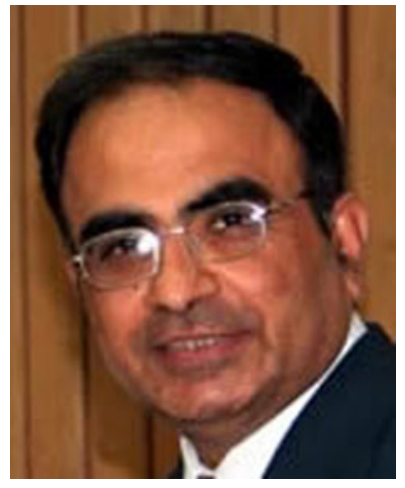

Soft tissue sarcomas are a heterogenous group of tumours that are notorious for recurrence. Since 1980s there has been a paradigm shift in the management of these cancers with the effort being more and more towards preservation of organ and function. This has become possible on account of a better understanding of the biological behaviour of these tumours. However there are still many grey areas that make these cancers unique. The cancers being rare and rarely diagnosed especially when located in deep organs, there are pitfalls in diagnosis and management. As opposed to "errors", pitfalls refer to unexpected or unforeseen difficulty or danger and do not include errors due to ignorance or lack of competence .Underestimation of both malignant potential and suboptimal surgical treatment of primary tumour are the major pitfalls that can be lethal in these cancers. [1-6]

Chintamani $(\triangle)$

VMMC, Safdarjang Hospital,

New Delhi 110023, India

e-mail: chintamani7@rediffmail.com
Biological Behaviour-Putting All Eggs in One Basket!!

It can be the most significant pitfall as this heterogeneous group behaves differently at different sites, the presentation and diagnosis could be confusing .Importantly, most pathologists have limited experience of studying these cancers on account of low incidence. With more than 20 histological subtypes it is nearly impossible for a single pathologist to encounter all subtypes in adequate numbers in his life time. Therefore mis/suboptimal diagnosis are a rule rather than an exception.

Inadequate or Inappropriate Biopsies (FNAC vs. Core Needle Biopsies vs. Incision Biopsies) and Sampling Errors [6-8]

Most centres have done away with FNAs (fine needle aspiration cytology) on account of false negatives and lack of ability to study the vital characteristics like grade that forms an integral part of staging in these cancers. FNA is mostly used to either make the initial diagnosis of exclusion or to ascertain the status of regional lymph nodes that have a major impact on the stage and outcome.

Regarding biopsies, the core needle biopsies (CNB) are usually optimum and the representative yield can be improved if these are done under image guidance especially in deep seated cancers. The only concern is the adequacy of tissue desired by the pathologist to make an optimum and detailed analysis.

Incision biopsies can yield more tissue; the pitfall lies in making wrong incisions (transverse incisions in the thigh or extremities etc.) that might influence the planning of incisions for the subsequent surgery. In small lesions that are located superficially there is no need to go for an overkill in terms of investigations, excision biopsies settle issues better provided clear all round margin is achievable. 
Sampling errors are additional pitfalls in the present era of tiny CT-guided needle biopsies.

\section{Inaccessible Sites or Wrong Site and Wrong Tumour at the Site}

Pitfalls often result on account of difficulties in diagnosis due to inaccessible sites, wrongly done biopsies or inadequate sample biopsy tissue and above all due to irrelevant and often excessive investigations.. There are therefore certain "grey areas" of ignorance that may lead to over or under interpretation of some morphologic or immune phenotypic features leading to misdiagnosis. [6-8]

\section{Benign Lesions that may be Confused as Malignant (Potential Pitfalls!)[8-12]}

The classical examples are

a. Cellular benign fibrous histiocytoma that shows central necrosis in as many as $10-15 \%$ of cases and also very often extends into underlying subcutaneous adipose tissue.

B. Atypical fibrous histiocytoma (so-called 'dermatofibroma with monster cells') is often mistaken for sarcoma, due to the presence of multifocally scattered large bizarre and often multinucleate cells with atypical hyperchromatic nuclei as well as mitotic figures which may be abnormal.

c. Lipoma showing extensive microscopic areas of fat necrosis is very frequently mistaken for atypical lipomatous tumour-however, such lesions entirely lack true adipocytic or stromal nuclear atypia or hyperchromasia.

A dedicated pathologist and immunohistochemical staining may help in making the diagnosis in these scenarios

\section{Malignant Lesions Mistaken as Benign [10-13]}

Low-grade fibromyxoid sarcoma is a prominent and the best-known example of this potential pitfall, although they have a characteristic translocation which can be detected by FISH or RT-PCR

\section{Histopathological Interpretation [High vs. low vs. Intermediate Grade Tumours]}

Histological grade is one of the most important predictors of outcome. Most pathologists would like to divide these tumours in to low and high grades depending on various factors that are based on morphologic diagnosis which may be subjective. Immunohistochemistry and, more recently, molecular methods along with ultra structure studies have further improved the sensitivity in the diagnosis of soft tissue sarcomas. This is another area of controversy as some pathologists would like to divide these tumours in to four categories with the second and third being intermediate groups. The division in to various grades can often be very subjective making the entire planning based on grades vulnerable

\section{Imaging [Can We bet on PET?]}

MRI is considered better than computed scan (CT scan) except perhaps in the abdomen (retroperitoneal sarcomas) as it shows the soft tissue planes better. Image guided biopsies can improve the sensitivity of CNB by improving the yield.

PET (positron emission tomography) scan still has an investigational role. In some studies it has been found to help identify the low grade from high grade tumours based on the take up activity. This can certainly be a more objective criteria that are based on the levels of activity of a particular tumour while ascertaining the grade. The most exciting role that PET can play is perhaps in the assessment of response to neo-adjuvant therapies (PERCIST-PET response evaluation criteria in solid tumours on the lines of RECIST -Response Evaluation Criteria in Solid Tumours). This is being used in some studies to highlight this role of PET in assessing response). The role may also be expanded to include assessment of recurrent tumours although it is still evolving and not a standard of care in soft tissue sarcomas [13-15]

\section{Pitfalls in the Management}

Optimum Surgery [6-9]

Surgery is the mainstay of management and there are enough studies to reveal that an optimum surgery in the form of R0 resection (microscopic freedom from cancer) does reduce the incidence of local recurrence and is the most important predictor of outcome in soft tissue sarcomas. Local recurrences have been found to be associated with higher rates of distant metastases and poorer outcome. The best chance that the patient has is in the setting of primary tumours. An optimum surgery with wide local excision including the scar of incisional biopsy is therefore the key. Poorly performed biopsy/primary surgery may jeopardize the chances of saving the life and/or a limb. 
It is now also well known that overtly aggressive local therapy (involving loss of an extremity) does not necessarily translate in to reduced local recurrence and distant failures influencing the overall survival in these cancers.

Various randomized controlled trials have successfully proven that the results of optimum local limb preserving surgery(R0 resection) followed by adjuvant radiotherapy are comparable to an excessively aggressive surgery involving loss of an organ(extremity).Therefore operations like "compartmentectomies" etc. are no longer common and popular. The bottom line is to have all round microscopically negative margins(R0). The common pitfalls in surgery are on account of an overzealous attempt to preserve the limb or the organs while compromising on the oncological safety. Technique of the surgeon and the surgeon himself could be a very important predictor of outcome and oncologically suboptimal surgery.

\section{Quality of Life -A Notion of Happiness}

In an overzealous attempt to provide a good quality of life, sometimes the surgeon may compromise on clear margins. There is no quality of life without life and one must address this issue with the patients' notion of happiness rather than deciding on what should make the patient's QOL better entirely from one's(surgeon's) own perspective. It is mandatory to have adequately wide (and deep margins) while appreciating that there are some natural barriers that can be exploited like the tendon sheaths, vascular sheaths etc.

\section{Radiotherapy and Chemotherapy (Pitfalls in Timing and Sequencing)}

The role of Radio and chemotherapy has now been established in these tumours but there are controversies regarding the timing or sequencing.

Pre-operative radiotherapy has the advantage in sighting the target better (as the tumour generally displaces the vital normal tissues away) reducing the collateral damage. The tissues (pre-surgery) are well oxygenated thus more responsive rather than post-surgery scarred tissues where the anatomy is distorted with reduced oxygenation. The radiotherapy if provided as Brachy therapy (BT) would help deliver a higher dose to the tumour bed with minimal collateral damage. However it is believed that the two (BT and EBRT) may be combined to achieve a higher dose delivery to the tumour bed while external beam radiotherapy (EBRT) may sterilize the margins better especially in larger tumours. The adjuvant radiotherapy is the standard of care in most centres and many trials have shown that $\mathrm{R} 0$ resection with adjuvant radiotherapy does reduce the incidence of local recurrence and survival in soft tissue sarcomas.
Preoperative chemotherapy has definite advantage in terms of control of micro metastases and assessing the in vivo chemo sensitivity of a particular regime so that the same regime may be used in the adjuvant setting. It may also help in downsizing the tumour thus allowing the clearance of the margins optimally. Response to neoadjuvant chemotherapy (NACT) is also an important indicator of the tumour behaviour and response predicts a favourable outcome.

These tumours continue to remain an enigma on account of the heterogenousity in addition to the limited experience at most centres and importantly lack of effective collaborative efforts between institutions. The most important pitfall is to generalize the management of these tumours as two histologically similar tumours behave differently at two different sites and would need different management.

There is thus a greater need to tailor the therapy to the tumour, site, patient, center and the facilities that are available. Saving life should take preference over the strongly felt notion of quality of life. It is better to have a living problem rather than a dead solution and therefore the bottom line in the management of these cancers is optimum ( $R 0$ resection) surgery, other modalities may contribute towards achieving this aim.

There can however be a difficulty achieving an R0 resection at certain anatomical sites e.g. retroperitoneal sarcomas. An effort should be made to preserve the adjoining vital organs. The more common ones i.e. "atypical lipomatous tumour" or the "well differentiated retroperitoneal soft tissue sarcomas" fortunately do well and do not metastasize unless there is dedifferentiation and R1 resection although not desirable may be acceptable in this subset.

Since these cancers are rare and difficult to diagnose and treat a collaborative effort from a team comprising of surgical oncologist, pathologist, radiologist, medical oncologist, and radiation oncologist is mandatory for a satisfactory outcome.

\section{References}

1. Heslin MJ, Lewis JJ, Woodruff JM et al (1997) Core needle biopsy for diagnosis of extremity soft tissue sarcoma. Ann Surg Oncol 4:425-431

2. Yao L, Nelson SD, Seeger LL et al (1999) Primary musculoskeletal neoplasms: effectiveness of core-needle biopsy. Radiology 212:682-686

3. Santucci M, Franchi A (2008) Recognizing hidden phenotypes in sarcomas through theelectron microscope. Ultrastruct Pathol 32:51-61

4. Huang H-Y, Antonescu CR (2002) Epithelioid variant of pleomorphic liposarcoma: a comparative immunohistochemical and ultrastructural analysis of six cases with emphasis on overlapping features with epithelial malignancies. Ultrastruct Pathol 26:299-308 
5. Erlandson RA (1994) Diagnostic Transmission Electron Microscopy of Tumors. Raven, New York

6. Lewis JJ, Leung D, Espat $\mathrm{J}$ et al (2000) Effect of reresection in extremity soft tissue sarcoma. Ann Surg 231:655-663

7. Eilber FC, Rosen G, Nelson SD et al (2003) High-grade extremity soft tissue sarcomas: factors predictive of local recurrence and its effect on morbidity and mortality. Ann Surg 237:218-226

8. Lewis JJ, Leung D, Heslin M et al (1997) Association of local recurrence with subsequent survival in extremity soft tissue sarcoma. J Clin Oncol 15:646-652

9. Eilber FC, Brennan MF, Riedel E, et al (2005) Prognostic factors for survival in patients with locally recurrent extremity soft tissue sarcomas. Ann Surg Oncol 942.

10. Fisher C (2006) The comparative roles of electron microscopy and immunohistochemistry in the diagnosis of soft tissue tumors. Histopathology 48:32-41
11. Tucker JA (2000) The continuing value of electron microscopy in surgical pathology. Ultrastruct Pathol 24:383-9

12. Herrera GA, Turbat-Herrera EA (2008) Sarcoma and look-alikes: the important role of ultrastructural evaluation. Ultrastruct Pathol $32: 43-50$

13. Alford S, Choong P, Chander S, Henderson M, Chu J, Powell G, Williams S, Ngan S (2011) Value of PET scan in patients with retroperitoneal sarcoma treated with preoperative radiotherapy. Eur J Surg Oncol.

14. Benz MR, Tchekmedyian N, Eilber FC, Federman N, Czernin J, Tap WD (2009) Utilization of positron emission tomography in the management of patients with sarcoma. Curr Opin Oncol 21 (4):345-51

15. Wahl RL, Jacene H, Kasamon Y, Lodge MA (2009) From RECIST to PERCIST: Evolving Considerations for PET response criteria in solid tumors. J Nucl Med 50(Suppl 1):122S-50S 